\title{
Quality of Life of Wheelchair Rugby Players
}

\author{
Tomáš Zelenka, Martin Kudláček, and Julie Wittmannová \\ Department of Adapted Physical Activity, Faculty of Physical Culture, Palacký University Olomouc, Czech Republic
}

\begin{abstract}
Quality of life (QOL) of persons with disabilities has recently become focus of studies in various academic disciplines There is $250-500$ thousands new cases of spinal cord injury annually, with majority being caused by accidents. Spinal cord injury (SCI) makes significant impact on person's lives as only one third of persons with SCI are able to return to jobs they did prior to acquiring SCI and majority of them cannot return to the hobbies or sports. The purpose of this study was to show the effect of sport participation in wheelchair rugby on QOL of persons with spinal cord injury. Twenty male wheelchair rugby players - athletes with tetraplegia, who are engages in regular organized physical activity - wheelchair rugby training and competition (mean age $=32.50, S D=8.46$ ). Sixteen persons with tetraplegia (all males) who were not actively engaging in sports - not participating in an organized physical activity (mean age $=39.44, S D=7.44$ ). While results of wheelchair rugby players were higher in all four domains of QOL, the only significant difference was found in domain one focusing on perceived physical health.
\end{abstract}

Keywords: Spinal cord injury, Health, Wellbeing, Perception

\section{Introduction}

Quality of life (QOL) of persons with disabilities has recently become focus of studies in various academic disciplines (Kawanishi \& Greguol, 2013). World Health Organization defines QOL as an individual's perception of their position in life in the context of the culture and value systems in which they live and in relation to their goals, expectations, standards and worries. It is a broad concept affected in a complex way by the person's physical health, psychological state, personal beliefs, social relationships and their relationship to salient features of their environment (World Health Organization, 2001). Recently there has been growing emphasis on QOL of persons with disabilities, moving away from the focus merely on their health conditions and impairments (Kawanishi \& Greguol, 2013).

There is $250-500$ thousands new cases of spinal cord injury annually, with majority (aprox. 90\%) being caused by accidents. Spinal cord injury (SCI) is closely connected with issues of participation in different activities. There is a decrease in occupational activities in persons with SCI (Schönherr, Groothoff, Mulder, \& Eisma, 2005), problematic return to jobs and significantly higher rates of unemployment (Krause, Terza, Saunders, \& Dismuke, 2010; McVeigh, Hitzig \& Craven, 2009; Schönherr et al., 2005; Tasiemski, Kennedy, Gardner \& Taylor, 2005). Persons with SCI have lower rate of participation in physical activities, lower rates in sport participation (Sale et al. 2012; Schönherr et al., 2005; Tasiemski, Kennedy, Gardner, \& Blaikley, 2004) and higher rates of sedentary activities such as reading, music listening, watching movies, PC games (Tasiemski et al., 2005). SCI makes significant impact on person's lives as only one third of persons with SCI are able to return to jobs they did prior to acquiring SCI (Krause et al., 2010) and majority of them cannot return to the hobbies (Schönherr et al., 2005) or sports (Tasiemski et al., 2004; Urbanski, Bauerfeind \& Pokaczajlo, 2013; Wandell, 2010) they did prior to SCI. McKinley and Meade (2004) described the ongoing long-term problems with participation in social activities among persons with SCI. Unfortunately, non-participation among persons with SCI has negative trend during the life after SCI (Charlifue \& Gerhart, 2004; Scelza et al., 2007). Lemay (1999) emphasizes that interpersonal interactions and experience with different social roles play crucial roles in creations of self-identity, self-esteem and ability to social adaptation.

Review studies on QOL, SCI and physical activities emphasize problems with methodological issues causing inconsistent findings (Ginis, Jetha, Mack \& Hetz, 2010; Hill, Noonan, Sakakibara, \& Miller, 2010; Kawanishi \& Greguol, 2013). Ginis with colleagues (2010) showed in meta-analytical study focused on physical activity, QOL and SCI, that physical activity closely correlates with subjective well-being and life satisfaction. Noce, Simim and Melo (2009) claim that regular 
physical activity can facilitate re-integration of persons with SCI after return from rehabilitation and ease overcoming emerging physical, social and psychological challenges. Important benefits of physical activity for persons with SCI are an increase in functional independency and improvement in activities of daily living (Dixon-Ibarra \& Driver, 2013), meaningful experiences helping with coping with post traumatic development (Day, 2013) and positive changes resulting from facing challenging life situations (Tedeschi \& Calhoun, 2004). Unfortunately, while positive effects of physical activity on lives of persons with SCI are certain (van der Ploeg, van der Beek, van der Woude, \& van Mechelen, 2004), their participation levels are lower than participation in nondisabled population (Kawanishi \& Greguol, 2013). The purpose of this study was to show the effect of sport participation in wheelchair rugby on QOL of persons with SCI.

\section{Methods}

\section{Sample}

Twenty male wheelchair rugby players - athletes with tetraplegia, who are engaged in regular organized physical activity - wheelchair rugby training and competition (mean age $=32.50, S D=8.46$ ). Sixteen persons with tetraplegia (all males) who were not actively engaged in sports - not participating in an organized physical activity (mean age $=39.44, S D=7.44$ ).

\section{Questionnaire WHOQOL-BREF}

The World Health Organization Quality of Life (WHOQOL) project was initiated in 1991 by a working group of 15 international research centres. The aim was to develop an international cross-culturally comparable QOL assessment instrument. It assesses the individual's perceptions in the context of their culture and value systems, and their personal goals, standards and concerns. The WHOQOL instruments were developed collaboratively in a number of centres worldwide, and have been widely field-tested. The WHOQOL-BREF instrument comprises of 26 items, which measure the following four domains:

a) physical health (DOM 1),

b) psychological health (DOM 2),

c) social relationships (DOM 3), and

d) environment (DOM 4).

First two items have general QOL (Q1) and general health (Q2). The WHOQOL-BREF is a shorter version of the original instrument (WHOQOL-100) that may be more convenient for use in large research studies or clinical trials. Czech version of WHOQOL-BREF instrument was prepared by a research Psychiatric centre in Prague (Dragomirecká \& Bartoňová, 2006).

\section{Administration and evaluation of WHOQOL-BREF}

WHOQOL-BREF instrument is self-administered and it takes about 5 minutes to complete. Items Q1 and Q2 are evaluated independently and are scored in Likert scale 1-5 with higher score representing higher QOL. WHOQOL-BREF then measures four domains. Physical health domain (DOM 1) consists of the following items: Activities of daily living; Dependence on medicinal substances and medical aids; Energy and fatigue; Mobility; Pain and discomfort; Sleep and rest; and Work Capacity. Psychological health domain (DOM 2) consists of the following items: Bodily image and appearance; Negative feelings; Positive feelings; Self-esteem; Spirituality/Religion/Personal beliefs; Thinking, learning, memory and concentration. Social relationships domain (DOM 3) consists of the following items: Personal relationships; Social support; and Sexual activity. Environmental domain (DOM 4) consists of the following items: Financial resources; Freedom, physical safety and security; Health and social care: accessibility and quality; Home environment; Opportunities for acquiring new information and skills; Participation in and opportunities for recreation/leisure activities; Physical environment (pollution/noise/traffic/climate); and Transport. Domain scores are arithmetical means of domain specific items multiplied by number 4 to allow comparability with WHOQOL-100 instrument. Domain specific scores range from 4 to 20 with higher scores representing higher QOL (Dragomirecká \& Bartoňová, 2006).

\section{Results}

There is a visible tendency to show higher perceived QOL among athletes - wheelchair rugby players in comparison with persons with similar levels of impairment, who do not live an active lifestyle. Although only one domain showed significant differences between athletes and non-athletes (Table 2). This difference was in domain 1 - Physical health. Athletes showed higher values in all items with greatest differences being in better QOL related to sleep and rest and lesser dependency on medical aids and services. In second domain - Psychological quality (Table 1) of life, athletes had higher scores on most items with two exceptions:

a) Bodily image and

b) Self-esteem. 
Table 1

Descriptive statistics for WHOQOL domains and individual items

\begin{tabular}{|c|c|c|c|c|}
\hline \multirow{2}{*}{$\begin{array}{l}\text { Domain } \\
\text { Items }\end{array}$} & \multicolumn{2}{|c|}{$\begin{array}{l}\text { Rugby players } \\
\qquad(n=20)\end{array}$} & \multicolumn{2}{|c|}{$\begin{array}{l}\text { Non-athletes } \\
\quad(n=16)\end{array}$} \\
\hline & $M$ & $S D$ & $M$ & $S D$ \\
\hline Physical health* & 14.60 & 1.85 & 12.93 & 2.26 \\
\hline Activities of daily living & 3.65 & 1.09 & 3.38 & 1.09 \\
\hline Dependence on medical aids & 4.20 & 0.62 & 3.69 & 1.08 \\
\hline Energy and fatigue & 3.50 & 0.76 & 3.44 & 1.03 \\
\hline Mobility & 3.21 & 0.92 & 2.69 & 1.08 \\
\hline Pain and discomfort & 3.55 & 1.15 & 3.31 & 0.87 \\
\hline Sleep and rest & 3.80 & 0.70 & 2.87 & 0.81 \\
\hline Work Capacity & 3.65 & 1.04 & 3.25 & 1.00 \\
\hline Psychological & 15.89 & 1.89 & 15.38 & 1.48 \\
\hline Bodily image and appearance & 4.05 & 0.76 & 4.13 & 0.81 \\
\hline Negative feelings & 3.85 & 0.99 & 3.69 & 0.95 \\
\hline Positive feelings & 4.00 & 0.80 & 3.75 & 1.00 \\
\hline Self-esteem & 4.11 & 0.57 & 4.44 & 0.81 \\
\hline Spirituality/Personal beliefs & 3.85 & 0.59 & 3.31 & 0.87 \\
\hline Thinking, learning,... & 4.00 & 0.65 & 3.69 & 1.08 \\
\hline Social relationships & 15.02 & 2.99 & 13.58 & 3.27 \\
\hline Personal relationships & 3.85 & 0.86 & 3.37 & 1.15 \\
\hline Social support & 3.40 & 0.94 & 2.44 & 1.21 \\
\hline Sexual activity & 4.02 & 1.14 & 4.38 & 0.81 \\
\hline Environment & 14.91 & 1.56 & 14.71 & 2.44 \\
\hline Financial resources & 3.90 & 0.72 & 3.69 & 1.08 \\
\hline Freedom, and security & 3.47 & 0.84 & 3.50 & 0.63 \\
\hline Health and social care: accessibility & 3.55 & 1.10 & 3.69 & 1.25 \\
\hline Home environment & 4.45 & 0.61 & 4.56 & 0.63 \\
\hline Opportunities for new information & 3.60 & 0.68 & 3.13 & 1.26 \\
\hline Opportunities for leisure activities & 3.75 & 0.79 & 3.87 & 0.74 \\
\hline Physical environment & 3.85 & 0.75 & 3.88 & 0.96 \\
\hline Transport & 3.25 & 1.02 & 3.19 & 1.17 \\
\hline General quality of life? & 3.95 & 0.51 & 3.56 & 0.89 \\
\hline Satisfaction with own health? & 3.30 & 0.92 & 2.94 & 1.12 \\
\hline
\end{tabular}

Note. $*=$ statistically significant difference $p<.05$ using Mann-Whitney U test.

Table 2

Mann-Whitney $U$ test

\begin{tabular}{|c|c|c|c|c|c|c|}
\hline & qlf1 & qlf2 & DOM 1 & DOM 2 & DOM 3 & DOM 4 \\
\hline Mann-Whitney U & 111.00 & 136.00 & 91.00 & 142.50 & 119.00 & 147.00 \\
\hline Z & -1.72 & -0.81 & -2.22 & -0.56 & -1.32 & -0.42 \\
\hline Asymp. Sig. (2-tailed) & 0.09 & 0.42 & $0.03 *$ & 0.57 & 0.19 & 0.68 \\
\hline
\end{tabular}

Note. $\quad$ DOM $1=$ physical health domain, DOM $2=$ psychological health domain, DOM $3=$ social relationships domain; DOM $4=$ Environmental domain; qlf1 = general quality of life; qlf2 = satisfaction with own health; $*$ = statistically significant difference $p<.05$ using Mann-Whitney U test. 
In the third domain on Social relationships, athletes scored higher on overall domain with higher scores on perceived social support but lower scores on sexual activity, which resulted in non-significant difference in this domain. Perception of environmental constrains was comparable in both groups. Athletes only scored higher in the item focused on opportunities to acquire new information or skills. Wheelchair rugby players scored higher on both general items having better perception about their overall QOL with 3.95 vs. 3.56 in non-athletes with quadriplegia. However, this difference was not significant with $p=0.09$. Athletes were better satisfied with their health with 3.30 vs. 2.94 in non-athletes, but also this difference was not statistically significant $(p=0.42)$.

\section{Discussion}

The greatest perceived benefits resulting from sport participation were found in domain Physical health. In the agreement with Dixon-Ibarra and Driver (2013), sport participation seems to increase functional independency and improvement in activities of daily living, there is a better mobility and work capacity and much lesser dependency on medical support and better sleep and rest, which can result in helping to cope with post traumatic development (Day, 2013). Social benefits emphasized by Lemay (1999) were also confirmed. Wheelchair rugby players are experiencing new interpersonal interactions and different social roles. This has tremendous impact on creations of self-identity, self-esteem and ability to social adaptation. The number of participants willing to take part in this study was limited. The sample was purposive as we targeted persons with comparable impairments and only one aspect of their life being significantly different sport-non/participation. Therefore we were facing many methodological limitations, as highlighted by Ginis with colleagues (2010). Even with these limitations we were able to find the positive effect of sport participation in the QOL related to physical health and perceptions of independency and greater mobility.

\section{Acknowledgment}

This study was supported by grant IGA FTK_2017_006 Monitoring of physical activity and lifestyle as crucial determinant of health and quality of persons with disabilities.

\section{References}

Dixon-Ibarra, A., \& Driver, S. (2013). The role of self-efficacy in physical activity participation for persons with disabilities. Palaestra, 27(4), 31-3.

Day, M. C. (2013). The role of initial physical activity experiences in promoting posttraumatic growth in Paralympic athletes with an acquired disability. Disability \& Rehabilitation, 35(24), 2064-2072.

Dragomirecká, E. \& Bartoňová, J. (2006). WHOQOL-BREF. WHOQOL-100. Přiručka pro uživatele české verze dotazníku kvality života Světové zdravotnické organizace. (WHOQOLBREF. WHOQOL-100. Guide of Czech Instrument on Quality of Life from World Health Organisation). Praha: Psychiatrické centrum Praha.

Ginis, K. M., Jetha, A., Mack, D. E., \& Hetz, S. (2010). Physical activity and subjective well-being among people with spinal cord injury: a meta-analysis. Spinal Cord, 48(1), 65-72.

Hill, M. R., Noonan, V. K., Sakakibara, B. M., \& Miller, W. C. (2010). Quality of life instruments and definitions in individuals with spinal cord injury: a systematic review. Spinal cord, 48(6), 438-450.

Charlifue, S., \& Gerhart, K. (2004). Community integration in spinal cord injury of long duration. NeuroRehabilitation, 19, 91-101.

Kawanishi, C. Y., \& Greguol, M. (2013). Physical activity, quality of life, and functional autonomy of adults with spinal cord injuries. Adapted Physical Activity Quarterly, 30(4), 317-337.

Krause, J. S., Terza, J. V., Saunders, L. L., \& Dismuke, C. E. (2010). Delayed entry into employment after spinal cord injury: factors related to time to first job. Spinal Cord, 48(6), 487-491.

Lemay, R. A. (1999). Roles, identities, and expectancies: positive contributions to normalization and social role valorization. A quarter-century of normalization and social role valorization: Evolution \& impact, 219-240.

McKinley, W. O., \& Meade, M. A. (2004). Community integration following SCI. NeuroRehabilitation, 19, 79-80.

McVeigh, S. A., Hitzig, S. L., \& Craven, B. C. (2009). Influence of sport participation on community integration and quality of life: a comparison between sport participants and nonsport participants with spinal cord injury. The journal of spinal cord medicine, 32(2), 115-124.

Noce, F., Simim, M. A. D. M., \& Mello, M. T. D. (2009). Can the self-perceived quality of life of people with special needs be influenced by practice of physical activity? Revista Brasileira de Medicina do Esporte, 15(3), 174-178.

Sale, P., Mazzarella, F., Pagliacci, M. C., Aito, S., Agosti, M., \& Franceschini, M. (2012). Sport, free time and hobbies in people with spinal cord injury. Spinal cord, 50(6), 452-456.

Scelza, W. M., Kirshblum, S. C., Wuermser, L. A., Ho, C. H., Priebe, M. M., \& Chiodo, A. E. (2007). Spinal cord injury medicine. 4. Community reintegration after spinal cord injury. Archives of physical medicine and rehabilitation, 88(3), S71-S75.

Schönherr, M. C., Groothoff, J. W., Mulder, G. A., \& Eisma, W. H. (2005). Participation and satisfaction after spinal cord injury: results of a vocational and leisure outcome study. Spinal cord, 43(4), 241-248. 
Tedeschi, R. G., \& Calhoun, L. (2004). Posttraumatic growth: a new perspective on psychotraumatology. Psychiatric Time, 21(4), 1-15.

Tasiemski, T., Kennedy, P., Gardner, B. P., \& Blaikley, R. A. (2004). Athletic identity and sports participation in people with spinal cord injury. Adapted Physical Activity Quarterly, 21(4), 364-378.

Tasiemski, T., Kennedy, P., Gardner, B. P., \& Taylor, N. (2005). The association of sports and physical recreation with life satisfaction in a community sample of people with spinal cord injuries. NeuroRehabilitation, 20(4), 253-265.

Urbanski, P., Bauerfeind, J., \& Pokaczajlo, J. (2013). Community integration in persons with spinal cord injury participating in team and individual sports. Trends in Sport Sciences, 20(2), 95-100.

van der Ploeg, H. P., van der Beek, A. J., van der Woude, L. H., \& van Mechelen, W. (2004). Physical activity for people with a disability. Sports Medicine, 34(10), 639-649.

Wandell, N. (2010). The effect of social participation on adults with spinal cord injuries.

World Health Organization. (2001). The International Classification of Functioning, Disability and Health - ICF. Geneva: World Health Organization. Retrieved from http://www.who. int/classifications/icf/en/

\section{Corresponding author}

Tomáš Zelenka

Email address | tomas.zelenka01@upol.cz 